\title{
Investigação sobre adesão à terapêutica na população portuguesa: uma revisão de âmbito
}

André Coelho, ${ }^{1}$ Cláudia Vilares, ${ }^{2}$ Mariana Silva,${ }^{3}$ Catarina Rodrigues, ${ }^{4}$ Marta Costa, ${ }^{5}$ Sara Gordicho, ${ }^{6}$ Pedro Caetano ${ }^{7}$

\section{RESUMO}

Objetivos: Realizar uma revisão sobre adesão à terapêutica na população portuguesa visando descrever o âmbito (quantidade, foco e natureza) da atividade de investigação original.

Fontes de dados: Foram pesquisados estudos sobre adesão à terapêutica na MEDLINE, Web of Science, SciELO e B-on.

Métodos de revisão: A revisão foi realizada em dezembro de 2014. Nas fontes de dados foram pesquisados estudos sobre adesão à terapêutica na população portuguesa cuja recolha de dados fosse igual ou posterior a 2005 e/ou se a publicação ocorreu até ao final de 2014.

Resultados: Obtiveram-se 82 publicações, das quais foram selecionadas 26 . Os resultados indicam um maior número de estudos em populações do Norte e da região de Lisboa e Vale do Tejo; a aparente inexistência de estudos sobre iniciação do tratamento e apenas um estudo sobre descontinuação; uma variabilidade nos métodos de recolha de dados e nas medidas utilizadas para avaliar a adesão à terapêutica, com os inquéritos dirigidos aos doentes como a principal fonte de informação; taxas de adesão entre os $41,6 \%$ e os $89 \%$, dependentes da doença ou condição estudada.

Conclusões: Os diferentes métodos de recolha de dados e medidas de avaliação da adesão, os curtos períodos de recolha de dados, a análise quase exclusiva da implementação do tratamento, não analisando os fatores que influenciam a sua iniciação e descontinuação, dificultam a monitorização da adesão à terapêutica na população portuguesa.

Palavras-chave: Adesão terapêutica; Revisão; Portugal

\section{INTRODUÇÃO}

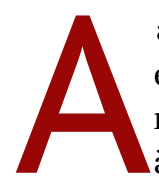
adesão à terapêutica é um fator-chave na gestão eficaz da doença na prática clínica, particularmente relevante nas doenças crónicas. A adesão à terapêutica assume-se como um determinante primário da efetividade do tratamento, uma vez que uma fraca adesão interfere com os esforços terapêuticos, reduzindo os benefícios clínicos da medicação e promovendo a utilização de meios de diagnóstico e tratamento desne-

1. Professor Adjunto. Área científica de Farmácia. Departamento das Ciências e Tecnologias Laboratoriais e Saúde Comunitária. Escola Superior de Tecnologia da Saúde de Lisboa, Instituto Politécnico de Lisboa.

2. Técnica de Farmácia. Farmácia da Misericórdia de Cuba.

3. Estudante do Mestrado em Engenharia Farmacêutica. Instituto Superior Técnico

e Faculdade de Farmácia da Universidade de Lisboa.

4. Técnica de Farmácia. Farmácia Ferraz.

5. Técnica de Farmácia. Farmácia Pinto Leal.

6. Técnica de Farmácia. Hospital Beatriz Ângelo.

7. Global Regulatory Affairs Director. Ipsen Biopharm. cessários. ${ }^{1-6}$ Num momento em que se estima que o impacto económico mundial das doenças crónicas em 2020 corresponda a $65 \%$ das despesas para a saúde em todo o mundo, ${ }^{6-7}$ a adesão à terapêutica representa um papel fundamental no processo de gestão da medicação. ${ }^{8}$

No entanto, estima-se que o grau de adesão às terapêuticas crónicas nos países desenvolvidos seja apenas de $50 \%$ e que nos países subdesenvolvidos ou em vias de desenvolvimento seja ainda menor. ${ }^{1,9}$ Este facto constitui um problema global que levanta fortes preocupações ao nível da saúde pública, por ser um fenómeno frequente, generalizado e independente da área terapêutica. ${ }^{1,10}$

De uma forma geral, a adesão à terapêutica refere-se ao processo pelo qual os doentes tomam os medicamentos de acordo com uma dada prescrição/recomendação médica, integrando três componentes distintas entre si: iniciação (também descrita na literatura como adesão pri- 
mária), implementação e descontinuação. ${ }^{2,10-11} \mathrm{O}$ termo adesão implica a noção de uma cooperação entre prescritor e doente desde o início do processo, distinguindo-se de outro termo usado na literatura - compliance - onde o papel do doente é entendido como sendo passivo e de obediência cega à prescrição/recomendação médica. ${ }^{2-3,6}$

O processo começa com a iniciação do tratamento, quando o doente toma a primeira dose do medicamento prescrito. A partir desse ponto ocorre a implementação do tratamento, sendo que nesta etapa o doente toma a medicação tendo em conta a posologia prescrita até à última dose. A descontinuação marca o fim do tratamento, não sendo tomadas mais doses posteriormente. $O$ período de tempo entre a iniciação e a descontinuação denomina-se persistência., ${ }^{1,1-14}$ Deste modo, a não-adesão pode ocorrer numa (ou em mais do que uma) das seguintes situações: atraso ou não-iniciação do tratamento, má implementação ou interrupção precoce do tratamento prescrito. .-2,10-14 $^{1}$

A adesão à terapêutica é habitualmente expressa como uma fração ou percentagem das doses prescritas e que foram realmente tomadas pelo doente durante um período específico de tempo. ${ }^{2-3}$

Diversos métodos têm sido utilizados para medir a adesão à terapêutica, incluindo (i) questionários dirigidos aos doentes/autorrelatos dos doentes; (ii) contagem de comprimidos; (iii) monitores eletrónicos de medicação; (iv) taxas de renovação de prescrições; (v) utilização de marcadores bioquímicos, entre outros. ${ }^{1-3,6,12}$ Os vários métodos apresentam vantagens e desvantagens, diferindo na sua validade, confiabilidade e sensibilidade.

Uma revisão de âmbito - scoping review em inglês corresponde a um processo de revisão de literatura sobre um determinado tema com vista à obtenção de uma imagem geral do conhecimento sobre o mesmo, ${ }^{15-16}$ identificando lacunas de conhecimento e/ou permitindo resumir os principais estudos existentes sobre esse tema. ${ }^{17}$ A principal diferença para uma revisão sistemática de literatura prende-se com o facto de que esta visa uma avaliação inicial da qualidade dos estudos a incluir na revisão e uma síntese quantitativa e qualitativa dos resultados, enquanto na revisão de âmbito se faz apenas uma síntese qualitativa do âmbito, métodos e resultados da atividade de investigação. ${ }^{18}$

Em Portugal, que seja do nosso conhecimento, não foi até agora publicado nenhum estudo onde se descreva o âmbito e resultados da atividade de investigação original sobre adesão à terapêutica na população portugue- sa. Essa descrição tem uma elevada importância, particularmente a nível dos cuidados de saúde primários, dado ser este o contexto de saúde no qual a maioria da população portuguesa é acompanhada. Adicionalmente, tendo em conta o conhecido impacto da adesão na efetividade dos tratamentos, ${ }^{4,7-8}$ torna-se necessário identificar lacunas de conhecimento que possam contribuir para definir prioridades de investigação.

\section{Objetivo}

O presente estudo tem como objetivo realizar uma revisão de âmbito sobre adesão à terapêutica na população portuguesa, visando descrever o âmbito (quantidade, foco e natureza) da atividade de investigação original.

\section{MÉTODOS}

Esta revisão de âmbito foi realizada em dezembro de 2014, simultaneamente por duas equipas de dois investigadores. Dois peritos, investigadores com experiência em saúde pública, epidemiologia e investigação em saúde, validaram as opções metodológicas.

A revisão de âmbito envolveu as seguintes fases (Figura 1) em concordância com a metodologia proposta por Arksey e O'Malley: ${ }^{18}$ (1) estabelecimento da pergunta de investigação; (2) pesquisa de estudos relevantes; (3) seleção dos estudos baseados nos critérios de inclusão pré-estabelecidos; (4) recolha de informação; e (5) resumo e comunicação da informação.

\section{Estratégias de pesquisa}

Foram incluídos estudos de adesão à terapêutica cujo ano de recolha de dados fosse igual ou posterior a 2005 e/ou se a publicação ocorreu entre janeiro de 2005 e dezembro de 2014 (janela temporal de 10 anos), quando o ano de recolha de dados fosse omisso. Foram excluídos estudos de revisão, por não constituírem investigação original, e/ou de validação de métodos de avaliação da adesão à terapêutica, bem como resumos de comunicações científicas orais e/ou sob a forma de póster em conferências.

As fontes de informação utilizadas foram a MEDLINE, a Web of Science, a SciELO e a biblioteca do conhecimento online (B-on).

Foram pesquisados estudos somente nas versões portuguesa e inglesa, combinando as palavras-chave através do operador booleano "AND". Na MEDLINE e na Web of Science utilizaram-se as palavras-chave 'medication adherence' AND 'Portugal'. Na SciELO e na B-on foram 


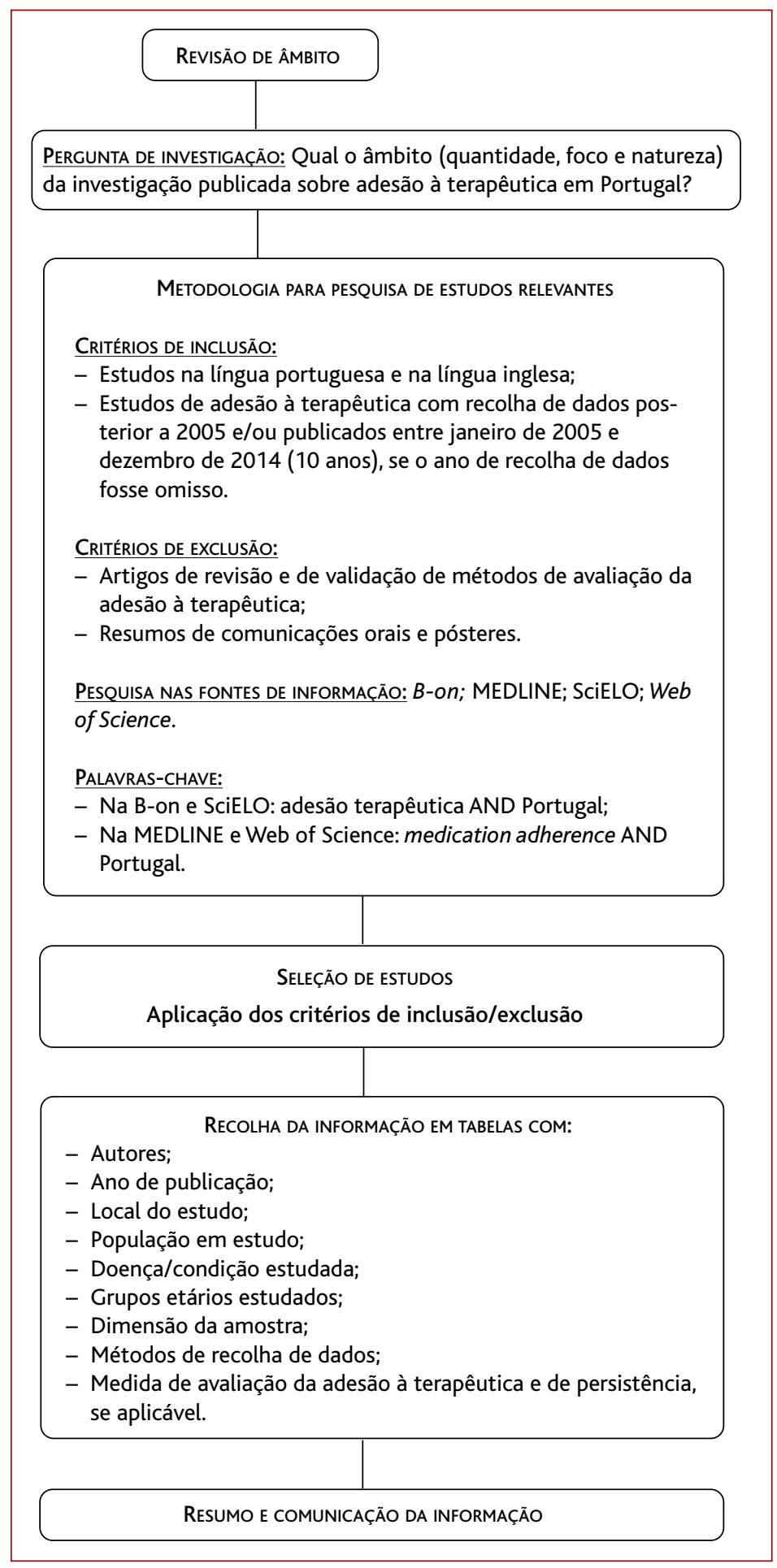

Figura 1. Metodologia adotada no processo de condução da revisão de âmbito. adicionalmente utilizadas as palavras-chave, em português, "adesão terapêutica" AND "Portugal".

A escolha da B-on e da SciELO com o uso das palavras-chave em português deveu-se à preocupação de incluir na análise dissertações de mestrado e teses de doutoramento, bem como estudos publicados em revistas portuguesas, já que a MEDLINE e a Web of Science não indexam todas as revistas publicadas em Portugal.

Nenhum critério de inclusão foi estabelecido quanto ao desenho de estudo ou grupos etários das populações em estudo. Não foi usada a qualidade dos estudos como critério de inclusão, como é recomendado nas revisões de âmbito. ${ }^{18}$

\section{Recolha da informação}

Foram recolhidos, numa tabela padrão, dados sobre as seguintes variáveis: autores, ano de publicação, local do estudo, população em estudo, doença/condição estudada, grupos etários estudados, dimensão da amostra, métodos de recolha de dados e medida de avaliação da adesão à terapêutica e de persistência, quando aplicável. Essa tabela foi preenchida à medida que os estudos foram selecionados, tornando posteriormente mais fácil a elaboração do resumo da informação quanto a: i) quantidade (número de estudos); ii) foco (populações, doenças/condições estudadas; grupos etários, métodos de recolha de dados e medidas de avaliação da adesão à terapêutica, entre outros); e iii) natureza (origem da atividade de investigação).

\section{RESULTADOS}

Obteve-se um total de 82 publicações, sendo excluídos da análise nove estudos de revisão/metaanálises; 11 estudos de validação de métodos de adesão à terapêutica, nomeadamente questionários, e 31 resumos de comunicações científicas em congressos. De referir que cinco artigos originais ${ }^{19-23}$ não foram considerados para análise, uma vez que resultaram de duas teses de doutoramento ${ }^{24-25} \mathrm{e}$ uma dissertação de mestrado ${ }^{26}$ também selecionadas para esta revisão, evitando, dessa forma, alguma redundância na análise (Figura 2). Desse modo, foram selecionadas para análise 26 publicações que satisfaziam os critérios de inclusão. 


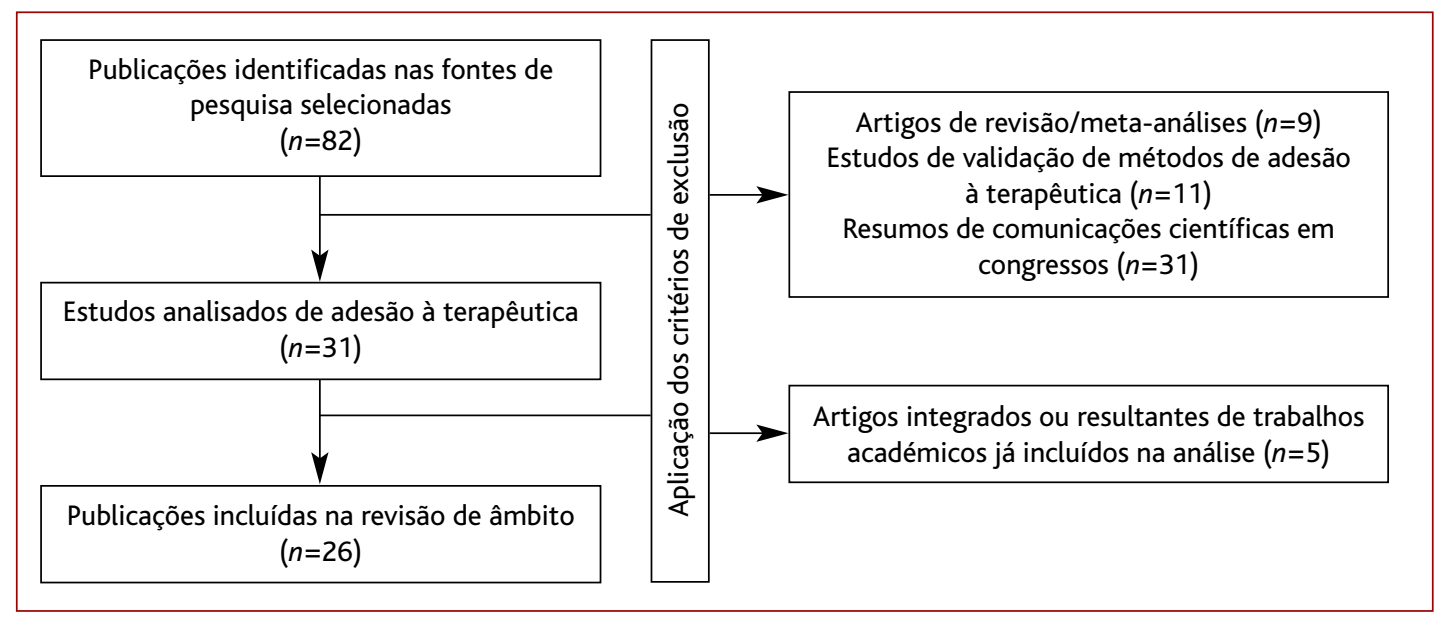

Figura 2. Diagrama para seleção das publicações analisadas.

O Quadro I apresenta os resultados de pesquisa de estudos originais sobre adesão à terapêutica em Portugal.

\section{Atividade de investigação sobre adesão à terapêutica em Portugal}

Os estudos selecionados sobre adesão à terapêutica são, na sua maioria, artigos originais - 15 no total (Quadro I) -, correspondendo os restantes a publicações académicas, nomeadamente duas teses de doutoramento ${ }^{24-25}$ e nove dissertações de mestrado..$^{26,32-36,48-49,51}$

\section{Local do estudo/Recolha dos dados}

Com a exceção do estudo de Margalho e colaboradores, ${ }^{27}$ todos os estudos têm âmbito regional ou local: sete na região Norte, ${ }^{26,32-33,36,41-42,49}$ sete na região de Lisboa eVale do Tejo, ${ }^{28,30,34,40,43-44,48}$ quatro na região Centro, ${ }^{25,35,45,51}$ dois no Alentejo ${ }^{31,37}$ e dois no Algarve. ${ }^{29,50} \mathrm{Em}$ dois dos estudos não é clara a região do País onde os mesmos decorreram;38-39 também o estudo de Reis $^{24}$ decorreu simultaneamente num hospital de Lisboa e num hospital do Porto.

Dos estudos selecionados, em 15 a amostra foi obtida a partir de utentes seguidos em consultas hospitalares de especialidade; ${ }^{24-27,29,32-35,38-39,42-43,48,51}$ em seis estudos, ${ }^{28,31,36-37,41,49}$ a partir de utentes seguidos nos cuidados de saúde primários - desde uma Unidade de Saúde Familiar no estudo de Chin e colaboradores ${ }^{28}$ até à Administração Regional de Saúde do Alentejo, no estudo de Moita; ${ }^{31}$ dois estudos recorreram a utentes de farmácias comunitárias; ${ }^{30,45} \mathrm{em}$ dois estudos, a amostra foi constituída por utilizadores de centros $\mathrm{de} \mathrm{dia}^{44,50} \mathrm{e}$, no último, por utentes seguidos na Associação Protetora dos Diabéticos de Portugal. ${ }^{40}$
Período de recolha de dados e tempo até publicação

A recolha de dados decorreu, em média, durante um período de 12 meses, período consideravelmente reduzido, principalmente tendo em conta a natureza crónica das doenças/condições estudadas. Em apenas seis estudos, ${ }^{24-26,313,36,40}$ a recolha de dados abrangeu um período igual ou superior a 12 meses, sendo nos restantes inferior - mínimo de um mês nos estudos de Chin e colaboradores ${ }^{28} \mathrm{e}$ de Monterroso e seus colaboradores. ${ }^{29}$ De referir, no entanto, que este período correspondeu, na maioria dos estudos, ao período de recrutamento dos doentes, sendo o período de análise da adesão à terapêutica propriamente dita consideravelmente menor. Em nove estudos, ${ }^{32,38-39,41-43,49-51}$ essa informação é omissa.

Verificou-se ainda um período considerável de tempo entre a recolha de dados e a publicação dos estudos, até um máximo de cinco anos no estudo de Fernandes e colaboradores..$^{30}$ Analisados os últimos dez anos de estudos originais publicados sobre adesão à terapêutica na população portuguesa, 19 das 26 publicações selecionadas ocorreram entre 2012 e 2014 (Quadro I), tendência crescente a analisar nos próximos anos.

\section{Doença/Condição estudada}

Relativamente à doença/condição objeto de estudo de adesão à terapêutica, em cinco estudos ${ }^{24,27,32-34}$ foi avaliada a adesão à terapêutica antirretroviral no tratamento da infeção pelo VIH e, em igual número de estudos, ${ }^{25,31,35-}$ ${ }^{37}$ a adesão à terapêutica anti-hipertensiva. A adesão à terapêutica no tratamento da disfunção eréctil foi estudada em duas publicações ${ }^{38-39}$ e em igual número para o 


\begin{tabular}{|c|c|c|c|c|c|}
\hline $\begin{array}{l}\text { Referência } \\
\text { bibliográfica }\end{array}$ & Tipo publicação & $\begin{array}{c}\text { Período de recolha } \\
\text { de dados }\end{array}$ & $\begin{array}{c}\text { Ano de } \\
\text { publicação }\end{array}$ & $\begin{array}{l}\text { Local do } \\
\text { estudo }\end{array}$ & Local de recolha dos dados \\
\hline Reis $^{33}$ & $\begin{array}{l}\text { Dissertação de } \\
\text { mestrado }\end{array}$ & Jun2006 - Fev2007 & 2007 & Porto & Hospital Joaquim Urbano \\
\hline Sousa et al. ${ }^{41}$ & Artigo original & Sem informação & 2008 & Porto & $\begin{array}{l}\text { Dois centros de saúde e um } \\
\text { hospital da área do Grande } \\
\text { Porto }\end{array}$ \\
\hline Lisboa $^{32}$ & $\begin{array}{l}\text { Dissertação de } \\
\text { mestrado }\end{array}$ & Não definido & 2009 & Guimarães & Centro Hospitalar do Alto Ave \\
\hline Sousa ${ }^{49}$ & $\begin{array}{l}\text { Dissertação de } \\
\text { mestrado }\end{array}$ & Sem informação & 2009 & $\begin{array}{l}\text { Braga e Viana } \\
\text { do Castelo }\end{array}$ & 7 Centros de saúde \\
\hline Margalho et al. ${ }^{27}$ & Artigo original & Set2007 - Jul2008 & 2011 & Todo o País & $\begin{array}{l}\text { Hospitais de referência e } \\
\text { instituições } \\
\text { não-governamentais de apoio a } \\
\text { doentes seropositivos }\end{array}$ \\
\hline Morgado ${ }^{25}$ & $\begin{array}{l}\text { Tese de } \\
\text { doutoramento }\end{array}$ & Jul2009 - Jun2010 & 2011 & Covilhã & Centro Hospitalar Cova da Beira \\
\hline Sousa et al. ${ }^{50}$ & Artigo original & Sem informação & 2011 & Olhão & $\begin{array}{l}\text { Centro de dia da Associação } \\
\text { Cultural e de Apoio Social de } \\
\text { Olhão }\end{array}$ \\
\hline Costa $^{51}$ & $\begin{array}{l}\text { Dissertação de } \\
\text { mestrado }\end{array}$ & Sem informação & 2012 & Coimbra & $\begin{array}{l}\text { Centro de Medicina e } \\
\text { Reabilitação da Região Centro - } \\
\text { Rovisco Pais }\end{array}$ \\
\hline Reis $^{24}$ & $\begin{array}{l}\text { Tese de } \\
\text { doutoramento }\end{array}$ & Fev2009 - Fev2010 & 2012 & Lisboa e Porto & $\begin{array}{l}\text { Hospital Joaquim Urbano e } \\
\text { Hospital Curry Cabral }\end{array}$ \\
\hline Carvalheira et al. ${ }^{39}$ & Artigo original & Sem informação & 2012 & $\begin{array}{l}\text { Sem } \\
\text { informação }\end{array}$ & Clínica de andrologia \\
\hline Fernandes $^{34}$ & $\begin{array}{l}\text { Dissertação de } \\
\text { mestrado }\end{array}$ & $\begin{array}{l}\text { Mai2010 - Out2010 } \\
\text { (análise: } 2005-2008 \text { ) }\end{array}$ & 2012 & Lisboa & Hospital de Santa Maria \\
\hline Moreno ${ }^{48}$ & $\begin{array}{l}\text { Dissertação de } \\
\text { mestrado }\end{array}$ & Mar2012 - Mai2012 & 2012 & Lisboa & Hospital Curry Cabral \\
\hline Pinto e José ${ }^{37}$ & Artigo original & Ago2011 - Set2011 & 2012 & Alcácer do Sal & Centro de saúde \\
\hline Moita $^{31}$ & Artigo original & 2010-2011 & 2012 & Alentejo & Região de Saúde do Alentejo \\
\hline Chin et al. ${ }^{28}$ & Artigo original & Abr2011 & 2012 & Loures & Unidade de saúde familiar \\
\hline Monterroso et al. ${ }^{29}$ & Artigo original & $\begin{array}{l}\text { 17Abr2011 - } \\
\text { 5Mai2011 }\end{array}$ & 2012 & Faro & $\begin{array}{l}\text { Consulta externa de psiquiatria } \\
\text { do Centro Hospitalar Barlavento } \\
\text { Algarvio }\end{array}$ \\
\hline Silva ${ }^{35}$ & $\begin{array}{l}\text { Dissertação de } \\
\text { mestrado }\end{array}$ & Mar2012 - Nov2012 & 2013 & Covilhã & Centro Hospitalar Cova da Beira \\
\hline
\end{tabular}




\begin{tabular}{|c|c|c|c|c|c|}
\hline $\begin{array}{l}\text { Referência } \\
\text { bibliográfica }\end{array}$ & Tipo publicação & $\begin{array}{c}\text { Período de recolha } \\
\text { de dados }\end{array}$ & $\begin{array}{c}\text { Ano de } \\
\text { publicação }\end{array}$ & $\begin{array}{l}\text { Local do } \\
\text { estudo }\end{array}$ & Local de recolha dos dados \\
\hline Salgado et al. ${ }^{44}$ & Artigo original & Mar2009 - Mai2009 & 2013 & Lisboa & $\begin{array}{l}\text { Centros de dia na área } \\
\text { metropolitana de Lisboa } \\
\text { (Amadora) }\end{array}$ \\
\hline Mendes ${ }^{26}$ & $\begin{array}{l}\text { Dissertação de } \\
\text { mestrado }\end{array}$ & $\begin{array}{l}\text { Recrutamento entre } \\
\text { Jan2011 - Jul2012 } \\
\text { ( } 2 \text { anos de } \\
\text { seguimento) }\end{array}$ & 2013 & Porto & Centro Hospitalar de São João \\
\hline Ribeiro ${ }^{36}$ & $\begin{array}{l}\text { Dissertação de } \\
\text { mestrado }\end{array}$ & Jul2011 - Ago2012 & 2013 & Porto & ACeS Porto Oriental \\
\hline Magalhães et al. ${ }^{42}$ & Artigo original & Sem informação & 2014 & Guimarães & Centro Hospitalar do Alto Ave \\
\hline Mosca et al. ${ }^{45}$ & Artigo original & Jan2011 - Abr2011 & 2014 & Sabugal & Farmácia comunitária \\
\hline Fernandes et al. ${ }^{30}$ & Artigo original & Fev2009 - Abr2009 & 2014 & Lisboa & $\begin{array}{l}52 \text { Farmácias comunitárias de } \\
\text { Lisboa }\end{array}$ \\
\hline Carvalheira et al..$^{38}$ & Artigo original & Sem informação & 2014 & $\begin{array}{l}\text { Sem } \\
\text { informação }\end{array}$ & Clínica de andrologia \\
\hline Serrabulho et al. ${ }^{40}$ & Artigo original & Abr2011-Abr2012 & 2014 & Lisboa & $\begin{array}{l}\text { Associação Protetora dos } \\
\text { Diabéticos de Portugal }\end{array}$ \\
\hline Gonçalves ${ }^{43}$ & $\begin{array}{l}\text { Dissertação de } \\
\text { mestrado }\end{array}$ & Sem informação & 2014 & Lisboa & $\begin{array}{l}\text { Centro Hospitalar Lisboa Norte } \\
\text { (Hospital de Santa Maria e } \\
\text { Hospital Pulido Valente) }\end{array}$ \\
\hline
\end{tabular}

Legenda: ACES=agrupamento de centros de saúde.

tratamento da diabetes ${ }^{40-41}$. As restantes publicações abrangeram condições díspares, como sejam a insuficiência cardíaca crónica, ${ }^{26}$ doença inflamatória intestinal, ${ }^{42}$ doenças respiratórias, ${ }^{43}$ entre outras. Em três publicações, ${ }^{28,44,49}$ a doença estudada não é particularizada.

\section{Grupos etários/Idade dos participantes dos estudos e dimensão da amostra}

Com a exceção do estudo de Magalhães e colaboradores, ${ }^{32}$ onde foram incluídos na amostra doentes com menos de 18 anos, nos restantes a população em estudo correspondeu exclusivamente à população adulta, especificamente a população idosa nos estudos de Salgado e colaboradores ${ }^{44}$ e de Mosca e seus colaboradores ${ }^{45}$ (Quadro I). Já no que diz respeito à dimensão das amostras, estas vão desde os 20 participantes no estudo de Lisboa $^{32}$ até aos 22.450 no estudo de Moita ${ }^{31}$ (Quadro II).
Métodos de recolha dos dados e medida de avaliação da adesão à terapêutica

Os estudos selecionados recorreram a diferentes métodos de recolha de dados e medidas de avaliação da adesão à terapêutica (Quadro II).

A maioria dos estudos (22) recorreu a questionários dirigidos aos doentes - previamente validados ou construídos especificamente para o estudo - para recolha dos dados sobre adesão à terapêutica, seja de forma presencial seja através do telefone. Em dois dos estudos realizados em contexto hospitalar, os dados foram recolhidos através da consulta de processos clínicos ${ }^{32}$ e dos registos de dispensa pelos serviços farmacêuticos hospitalares.$^{34}$ No estudo de Moita, ${ }^{31}$ a adesão foi avaliada pela consulta dos dados de prescrição e levantamento de medicação nas farmácias comunitárias e no estudo de Men$\operatorname{des}^{26}$ recorreram-se a monitores eletrónicos de medica- 


\begin{tabular}{|c|c|c|c|c|}
\hline $\begin{array}{l}\text { Referência } \\
\text { bibliográfica }\end{array}$ & População em estudo & $\begin{array}{l}\text { Doença/Condição } \\
\text { estudada }\end{array}$ & $\begin{array}{l}\text { Grupos } \\
\text { etários/Idade } \\
\text { (em anos) }\end{array}$ & $\begin{array}{l}\text { Dimensão } \\
\text { da amostra }\end{array}$ \\
\hline $\operatorname{Reis}^{33}$ & $\begin{array}{l}\text { Doentes diagnosticados com infeção pelo VIH/SIDA, } \\
\text { que frequentem a consulta externa de infecciologia, } \\
\text { que utilizem antirretrovirais }\end{array}$ & Infeção VIH/SIDA & $20-68$ & 125 \\
\hline Sousa et al. ${ }^{41}$ & $\begin{array}{l}\text { Doentes com diabetes tipo } 2 \text { há mais de } 12 \text { meses, } \\
\text { com idade } \geq 30 \text { anos, com uma consulta de } \\
\text { enfermagem no último ano }\end{array}$ & Diabetes & $\begin{array}{l}\geq 30 \text { (idade } \\
\text { média: 62,6) }\end{array}$ & 212 \\
\hline Lisboa $^{32}$ & $\begin{array}{l}\text { Doentes diagnosticados com infeção pelo VIH/SIDA, } \\
\text { seguidos na consulta de doenças infecciosas e serviço } \\
\text { de ambulatório }\end{array}$ & Infeção VIH/SIDA & $\geq 20$ & 20 \\
\hline Sousa ${ }^{49}$ & $\begin{array}{l}\text { Doentes seguidos na rede de cuidados de saúde } \\
\text { primários }\end{array}$ & Sem informação & $18-73$ & 207 \\
\hline Margalho et al. ${ }^{27}$ & Doentes diagnosticados com infeção pelo VIH/SIDA & Infeção VIH/SIDA & $\begin{array}{r}\text { Idade média: } \\
\qquad 41,16\end{array}$ & 762 \\
\hline Morgado ${ }^{25}$ & $\begin{array}{l}\text { Doentes, adultos, com diagnóstico de HTA, que } \\
\text { acorreram à consulta de HTA/dislipidemia }\end{array}$ & $\begin{array}{l}\text { Hipertensão } \\
\text { Arterial }\end{array}$ & $\begin{array}{r}\text { Idade média: } \\
60 \pm 12\end{array}$ & 197 \\
\hline Sousa et al. ${ }^{50}$ & $\begin{array}{l}\text { Doentes, idade } \geq 60 \text { anos, autónomos, polimedicados } \\
\text { (pelo menos quatro medicamentos) com pelo menos } \\
\text { uma patologia diagnosticada }\end{array}$ & Polimedicação & $\geq 60$ & 51 \\
\hline Costa $^{51}$ & $\begin{array}{l}\text { Doentes com lesão medular, em contexto hospitalar } \\
\text { de reabilitação, com quadro clínico de tetraplegia ou } \\
\text { paraplegia }\end{array}$ & Lesão medular & $21-79$ & 42 \\
\hline Reis $^{24}$ & $\begin{array}{l}\text { Doentes diagnosticados com infeção pelo VIH/SIDA, } \\
\text { que frequentem a consulta externa de infecciologia, } \\
\text { que utilizem antirretrovirais }\end{array}$ & Infeção VIH/SIDA & $19-81$ & 295 \\
\hline Carvalheira et al. ${ }^{39}$ & $\begin{array}{l}\text { Doentes do sexo masculino com disfunção eréctil } \\
\text { tratados com inibidores da fosfodiesterase } 5\end{array}$ & Disfunção eréctil & $25-81$ & 300 \\
\hline Fernandes $^{34}$ & $\begin{array}{l}\text { Doentes diagnosticados com infeção pelo VIH/SIDA, } \\
\text { que frequentem a consulta externa de infecciologia, } \\
\text { com pelo menos um levantamento de antirretrovirais } \\
\text { entre } 01-01-2005 \text { e } 31-12-2008\end{array}$ & Infeção VIH/SIDA & $\begin{array}{l}\text { Idade média: } \\
\qquad 39,7 \pm 9,5\end{array}$ & 186 \\
\hline Moreno 48 & $\begin{array}{l}\text { Doentes submetidos a transplante renal ou hepático } \\
\text { há mais de seis meses }\end{array}$ & $\begin{array}{l}\text { Transplante renal/ } \\
\text { /hepático }\end{array}$ & $\geq 18$ & 75 \\
\hline Pinto e José ${ }^{37}$ & $\begin{array}{l}\text { Doentes hipertensos medicados, com consultas } \\
\text { regulares na instituição }\end{array}$ & $\begin{array}{l}\text { Hipertensão } \\
\text { arterial }\end{array}$ & $\geq 18$ & 61 \\
\hline Moita $^{31}$ & $\begin{array}{l}\text { Utentes residentes na Região de Saúde do Alentejo e } \\
\text { que entre } 2010 \text { e } 2011 \text { tivessem associadas pelo } \\
\text { menos duas prescrições de anti-hipertensivos, } \\
\text { especificamente ARAs }\end{array}$ & Hipertensão & $\geq 18$ & 22.450 \\
\hline
\end{tabular}




\begin{tabular}{|c|c|c|c|c|}
\hline $\begin{array}{l}\text { Referência } \\
\text { bibliográfica }\end{array}$ & População em estudo & $\begin{array}{l}\text { Doença/Condição } \\
\text { estudada }\end{array}$ & $\begin{array}{l}\text { Grupos } \\
\text { etários/ldade } \\
\text { (em anos) }\end{array}$ & $\begin{array}{l}\text { Dimensão } \\
\text { da amostra }\end{array}$ \\
\hline Chin et al. ${ }^{28}$ & $\begin{array}{l}\text { Utentes inscritos na USF, com médico de família, com } \\
\text { mais de } 17 \text { anos em abril de } 2011\end{array}$ & Sem informação & $\geq 18$ & 227 \\
\hline Monterroso et al. ${ }^{29}$ & $\begin{array}{l}\text { Doentes portadores de transtornos psiquiátricos, que } \\
\text { comparecem na consulta entre } 17 \text { de abril e } 5 \text { de } \\
\text { maio de } 2011 \text {, com vontade e capacidade de } \\
\text { participação no estudo }\end{array}$ & $\begin{array}{l}\text { Transtornos } \\
\text { psiquiátricos }\end{array}$ & $24-76$ & 61 \\
\hline Silva ${ }^{35}$ & $\begin{array}{l}\text { Doentes, adultos, com diagnóstico de HTA, } \\
\text { acompanhados durante, pelo menos, seis meses na } \\
\text { consulta de HTA/dislipidemia }\end{array}$ & $\begin{array}{l}\text { Hipertensão } \\
\text { arterial }\end{array}$ & $31-94$ & 101 \\
\hline Salgado et al. ${ }^{44}$ & Idosos, utilizadores de centros de dia & Sem informação & $\begin{array}{r}\text { Idade média: } \\
73,3\end{array}$ & 100 \\
\hline Mendes $^{26}$ & $\begin{array}{l}\text { Doentes diagnosticados com IC Crónica, sem } \\
\text { hospitalizações por IC nos três meses anteriores, com } \\
\text { prescrição farmacológica que incluísse } \\
\text { simultaneamente um IECA, bloqueador beta e } \\
\text { diurético da ansa }\end{array}$ & $\begin{array}{l}\text { Insuficiência } \\
\text { cardíaca crónica }\end{array}$ & $\begin{array}{r}\text { Idade média: } \\
63,5 \pm 13,2\end{array}$ & 63 \\
\hline Ribeiro $^{36}$ & $\begin{array}{l}\text { Doentes diagnosticados com HTA que frequentem as } \\
\text { consultas de hipertensão }\end{array}$ & $\begin{array}{l}\text { Hipertensão } \\
\text { arterial }\end{array}$ & $31-94$ & 117 \\
\hline Magalhães et al. ${ }^{42}$ & $\begin{array}{l}\text { Doentes diagnosticados com doença inflamatória } \\
\text { intestinal seguidos no departamento de } \\
\text { gastrenterologia }\end{array}$ & $\begin{array}{l}\text { Doença } \\
\text { inflamatória } \\
\text { intestinal }\end{array}$ & $16-75$ & 138 \\
\hline Mosca et al..$^{45}$ & $\begin{array}{l}\text { Doentes, idade } \geq 65 \text { anos, autónomos, que utilizem } \\
\text { três ou mais medicamentos, seguidos na farmácia em } \\
\text { relação ao perfil lipídico, glicémia ou pressão arterial }\end{array}$ & Polimedicação & $68-78$ & 54 \\
\hline Fernandes et al. ${ }^{30}$ & $\begin{array}{l}\text { Doentes com prescrição de um antibiótico oral para } \\
\text { tratamento de curta duração (<30 dias) }\end{array}$ & Infeção & $\begin{array}{r}\text { Idade média: } \\
46,5 \pm 16,6\end{array}$ & 243 \\
\hline Carvalheira et al. ${ }^{38}$ & $\begin{array}{l}\text { Doentes do sexo masculino com disfunção eréctil } \\
\text { tratados com inibidores da fosfodiesterase } 5\end{array}$ & Disfunção eréctil & $25-81$ & 327 \\
\hline Serrabulho et al. ${ }^{40}$ & $\begin{array}{l}\text { Doentes com diabetes tipo } 1 \text { que frequentaram } \\
\text { consultas de vigilância periódica entre abril de } 2011 \\
\text { e abril de } 2012\end{array}$ & Diabetes & $18-35$ & 278 \\
\hline Gonçalves ${ }^{43}$ & $\begin{array}{l}\text { Doentes respiratórios crónicos, a fazer tratamento há } \\
\text { pelo menos quatro meses }\end{array}$ & $\begin{array}{l}\text { Doença respiratória } \\
\text { (DPOC; síndroma } \\
\text { de apneia do sono } \\
\text { e asma) }\end{array}$ & $18-89$ & 166 \\
\hline
\end{tabular}

Legenda: VIH/SIDA=vírus da imunodeficiência humana/síndroma da imunodeficiência humana adquirida; IC=insuficiência cardíaca; HTA=hipertensão arterial; IECA=inibidor da enzima de conversão de angiotensina; DPOC=doença pulmonar obstrutiva crónica; ARAs=antagonistas dos recetores de angiotensina. 


\begin{tabular}{|c|c|c|c|c|}
\hline $\begin{array}{l}\text { Referência } \\
\text { bibliográfica }\end{array}$ & $\begin{array}{l}\text { Método de recolha } \\
\text { dos dados }\end{array}$ & $\begin{array}{l}\text { Outcomes } \\
\text { avaliados }\end{array}$ & $\begin{array}{c}\text { Medida de avaliação } \\
\text { da adesão à terapêutica } \\
\text { (quando aplicável) }\end{array}$ & Principais resultados \\
\hline Reis $^{33}$ & $\begin{array}{l}\text { Inquérito por } \\
\text { questionário } \\
\text { (CEAT-VIH) }\end{array}$ & $\begin{array}{l}\text { Adesão e } \\
\text { variáveis } \\
\text { psicológicas }\end{array}$ & $\begin{array}{l}\text { Pontuação obtida } \\
\text { no questionário }\end{array}$ & $\begin{array}{l}\text { Correlações importantes entre a } \\
\text { medida de adesão, a sintomatologia } \\
\text { emocional e a qualidade de vida }\end{array}$ \\
\hline Sousa et al. ${ }^{41}$ & $\begin{array}{l}\text { Inquérito por } \\
\text { questionário }\end{array}$ & $\begin{array}{l}\text { Adesão e } \\
\text { satisfação com } \\
\text { os cuidados de } \\
\text { enfermagem }\end{array}$ & $\begin{array}{l}\text { Pontuação obtida no } \\
\text { questionário }\end{array}$ & $\begin{array}{l}\text { Satisfação do utente relaciona-se com } \\
\text { maior adesão ao regime terapêutico }\end{array}$ \\
\hline Lisboa $^{32}$ & $\begin{array}{l}\text { Consulta de } \\
\text { processos clínicos e } \\
\text { registos de dispensa } \\
\text { pelos serviços } \\
\text { farmacêuticos }\end{array}$ & Adesão & $\begin{array}{l}\text { Frequência das aquisições } \\
\text { e tempo para dispensa } \\
\text { pelos serviços } \\
\text { farmacêuticos }\end{array}$ & $\begin{array}{l}\text { Utentes aderentes: alterações menos } \\
\text { frequentes do regime terapêutico; } \\
\text { supressão virológica máxima e } \\
\text { aumento do número de linfócitos T } \\
\text { CD4 }\end{array}$ \\
\hline Sousa ${ }^{49}$ & $\begin{array}{l}\text { Inquérito por } \\
\text { questionário (escala } \\
\text { de adesão } \\
\text { terapêutica) }\end{array}$ & $\begin{array}{l}\text { Adesão e } \\
\text { crenças }\end{array}$ & $\begin{array}{l}\text { Pontuação obtida no } \\
\text { questionário }\end{array}$ & $\begin{array}{l}\text { Quanto mais anos de } \\
\text { acompanhamento médico do utente } \\
\text { pelo médico de família, melhor é a } \\
\text { aliança médico-paciente e a adesão } \\
\text { terapêutica }\end{array}$ \\
\hline Margalho et al. ${ }^{27}$ & $\begin{array}{l}\text { Questionário de } \\
\text { autopreenchimento }\end{array}$ & $\begin{array}{l}\text { Adesão e } \\
\text { qualidade de } \\
\text { vida }\end{array}$ & $\begin{array}{l}\text { Adesão reportada pelo } \\
\text { doente }\end{array}$ & $\begin{array}{l}\text { - 82,5\% dos doentes referiram } \\
\text { cumprir integralmente as } \\
\text { prescrições médicas } \\
\text { - Doentes do grupo não-adesão } \\
\text { apresentaram piores resultados de } \\
\text { qualidade de vida em } 4 \text { dos } 6 \\
\text { domínios }\end{array}$ \\
\hline Morgado 25 & $\begin{array}{l}\text { Inquérito por } \\
\text { questionário (escala } \\
\text { de adesão à } \\
\text { terapêutica de } \\
\text { Morisky) }\end{array}$ & Adesão (taxa) & $\begin{array}{l}\text { Score obtido no } \\
\text { questionário }\end{array}$ & $\begin{array}{l}\text { Taxa de adesão: } 48,2 \% \\
\text { Taxa de controlo tensional: } 33 \%\end{array}$ \\
\hline Sousa et al. ${ }^{50}$ & $\begin{array}{l}\text { Inquérito por } \\
\text { questionário (escala } \\
\text { de adesão à } \\
\text { terapêutica de } \\
\text { Morisky) }\end{array}$ & Adesão (taxa) & $\begin{array}{l}\text { Score obtido no } \\
\text { questionário }\end{array}$ & $\begin{array}{l}\text { Taxa de adesão: } 45 \% \text { e } 49 \% \text { aderem } \\
\text { bastante ou completamente, } \\
\text { respetivamente, à terapêutica } \\
\text { instituída }\end{array}$ \\
\hline Costa $^{51}$ & $\begin{array}{l}\text { Inquérito por } \\
\text { questionário (escala } \\
\text { de adesão } \\
\text { terapêutica) }\end{array}$ & $\begin{array}{l}\text { Adesão e } \\
\text { variáveis } \\
\text { psicológicas }\end{array}$ & $\begin{array}{l}\text { Pontuação obtida no } \\
\text { questionário }\end{array}$ & $\begin{array}{l}\text { Sem diferenças significativas entre } \\
\text { aderentes e não-aderentes, exceto na } \\
\text { idade e da satisfação com suporte } \\
\text { social }\end{array}$ \\
\hline
\end{tabular}

ção (MEMS® - medication event monitoring system).

A pontuação ou score obtido nos questionários foi a medida de avaliação da adesão à terapêutica mais utilizada, permitindo a diferenciação entre níveis de adesão. 


\begin{tabular}{|c|c|c|c|c|}
\hline $\begin{array}{l}\text { Referência } \\
\text { bibliográfica }\end{array}$ & $\begin{array}{l}\text { Método de recolha } \\
\text { dos dados }\end{array}$ & $\begin{array}{l}\text { Outcomes } \\
\text { avaliados }\end{array}$ & $\begin{array}{c}\text { Medida de avaliação } \\
\text { da adesão à terapêutica } \\
\text { (quando aplicável) }\end{array}$ & Principais resultados \\
\hline Reis $^{24}$ & $\begin{array}{l}\text { Inquérito por } \\
\text { questionário } \\
\text { (CEAT-VIH) }\end{array}$ & $\begin{array}{l}\text { Adesão e } \\
\text { variáveis } \\
\text { psicológicas }\end{array}$ & $\begin{array}{l}\text { Pontuação obtida no } \\
\text { questionário }\end{array}$ & $\begin{array}{l}\text { Diferenças de adesão em variáveis } \\
\text { sociodemográficas (idade, ocupação e } \\
\text { história de toxicodependência) e } \\
\text { clínicas (via de transmissão, estado } \\
\text { serológico, marcadores biológicos, } \\
\text { tempo de tratamento, efeitos } \\
\text { secundários do tratamento, } \\
\text { interrupção e regime de tratamento) }\end{array}$ \\
\hline Carvalheira et al. ${ }^{39}$ & $\begin{array}{l}\text { Inquérito por } \\
\text { telefone }\end{array}$ & $\begin{array}{l}\text { Descontinuação } \\
\text { (taxa) }\end{array}$ & $\begin{array}{l}\text { Pontuação obtida no } \\
\text { questionário }\end{array}$ & $\begin{array}{l}48,9 \% \text { dos doentes descontinuaram } \\
\text { tratamento, } 55,1 \% \text { dos quais nos três } \\
\text { meses após início do tratamento }\end{array}$ \\
\hline Fernandes $^{34}$ & $\begin{array}{l}\text { Consulta de } \\
\text { processos clínicos e } \\
\text { registos de dispensa } \\
\text { pelos serviços } \\
\text { farmacêuticos }\end{array}$ & $\begin{array}{l}\text { Adesão } \\
\text { (qualidade da } \\
\text { execução) }\end{array}$ & $\begin{array}{l}\text { Medication possession } \\
\text { ratio e medication GAPs } \\
\text { (intervalos sem } \\
\text { medicação) }\end{array}$ & $\begin{array}{l}25,9 \% \text { dos doentes classificados } \\
\text { como não-aderentes no final do } \\
\text { período de estudo } \\
81,6 \% \text { dos não-aderentes tiveram } \\
\text { mais de um intervalo sem medicação } \\
\text { com duração < } 30 \text { dias }\end{array}$ \\
\hline Moreno $^{48}$ & $\begin{array}{l}\text { Inquérito por } \\
\text { questionário }\end{array}$ & Adesão & $\begin{array}{l}\text { Pontuação obtida no } \\
\text { questionário }\end{array}$ & $\begin{array}{l}44 \% \text { referem já terem esquecido de } \\
\text { tomar os seus medicamentos, embora } \\
\text { apenas } 9,3 \% \text { o tenham feito no mês } \\
\text { que antecedeu a recolha de dados }\end{array}$ \\
\hline Pinto e José ${ }^{37}$ & $\begin{array}{l}\text { Inquérito por } \\
\text { questionário (escala } \\
\text { de adesão à } \\
\text { terapêutica de } \\
\text { Morisky) }\end{array}$ & Adesão (taxa) & $\begin{array}{l}\text { Score obtido no } \\
\text { questionário }\end{array}$ & $\begin{array}{l}\text { Taxa de adesão: } 50 \% \text { com score } \geq 5,14 \\
\text { (média) }\end{array}$ \\
\hline Moita ${ }^{31}$ & $\begin{array}{l}\text { Consulta de registos } \\
\text { de prescrição e de } \\
\text { conferência de } \\
\text { receituário }\end{array}$ & Adesão & $\begin{array}{l}\text { Rácio de adesão primária, } \\
\text { MPR, Compliance Rate, } \\
\text { Refill Compliance Rate, } \\
\text { Continuous Measure of } \\
\text { Medication Gaps }\end{array}$ & $\begin{array}{l}\text { Adesão primária: } 0,612 \pm 0,325 ; \text { MPR: } \\
0,557 \pm 0,380 \text {; Compliance Rate: } \\
0,697 \pm 0,517 \text {; Refill Compliance Rate: } \\
0,695 \pm 0,518 \text {; Continuous Measure of } \\
\text { Medication Gaps: } 0,648 \pm 0,351\end{array}$ \\
\hline Chin et al. ${ }^{28}$ & $\begin{array}{l}\text { Inquérito por } \\
\text { questionário }\end{array}$ & Adesão & $\begin{array}{l}\text { Pontuação obtida no } \\
\text { questionário }\end{array}$ & $\begin{array}{l}79 \% \text { dos inquiridos referem não } \\
\text { cumprir a medicação - } 51 \% \text { por } \\
\text { razões financeiras }\end{array}$ \\
\hline Monterroso et al. ${ }^{29}$ & $\begin{array}{l}\text { Inquérito por } \\
\text { questionário (MAT) }\end{array}$ & Adesão & $\begin{array}{l}\text { Score obtido no } \\
\text { questionário }\end{array}$ & $\begin{array}{l}50,8 \% \text { aderem ao regime terapêutico } \\
\text { - níveis de adesão mais baixos nos } \\
\text { indivíduos mais velhos, com nível de } \\
\text { escolaridade mais baixo e classe social } \\
\text { mais baixa }\end{array}$ \\
\hline
\end{tabular}




\begin{tabular}{|c|c|c|c|c|}
\hline $\begin{array}{l}\text { Referência } \\
\text { bibliográfica }\end{array}$ & $\begin{array}{l}\text { Método de recolha } \\
\text { dos dados }\end{array}$ & $\begin{array}{l}\text { Outcomes } \\
\text { avaliados }\end{array}$ & $\begin{array}{l}\text { Medida de avaliação } \\
\text { da adesão à terapêutica } \\
\text { (quando aplicável) }\end{array}$ & Principais resultados \\
\hline Silva ${ }^{35}$ & $\begin{array}{l}\text { Inquérito por } \\
\text { questionário (escala } \\
\text { de adesão à } \\
\text { terapêutica de } \\
\text { Morisky) }\end{array}$ & Adesão (taxa) & $\begin{array}{l}\text { Score obtido no } \\
\text { questionário }\end{array}$ & $\begin{array}{l}\text { Taxa de adesão: } 41,6 \% \text { - os doentes } \\
\text { com a pressão arterial controlada } \\
\text { apresentavam uma taxa de adesão à } \\
\text { medicação significativamente mais } \\
\text { elevada }\end{array}$ \\
\hline Salgado et al. ${ }^{44}$ & $\begin{array}{l}\text { Inquérito por } \\
\text { questionário (MAT) }\end{array}$ & $\begin{array}{l}\text { NVS e Adesão } \\
\text { (taxa) }\end{array}$ & $\begin{array}{l}\text { Score obtido no } \\
\text { questionário }\end{array}$ & Sem relação entre o NVS e MAT \\
\hline Mendes $^{26}$ & $\begin{array}{l}\text { Dispositivos } \\
\text { eletrónicos de } \\
\text { monitorização } \\
\text { (MEMS) }\end{array}$ & Adesão (taxa) & $\begin{array}{l}\text { Percentagem de } \\
\text { cumprimento de doses } \\
\text { prescritas durante o } \\
\text { período de monitorização }\end{array}$ & $\begin{array}{l}\text { A proporção de tomas cumpridas foi } \\
\text { tendencialmente elevada para os três } \\
\text { fármacos, mas para cada fármaco } \\
\text { entre } 20 \% \text { a } 30 \% \text { dos doentes } \\
\text { cumpriram menos de } 88 \% \text { das doses } \\
\text { prescritas }\end{array}$ \\
\hline Ribeiro ${ }^{36}$ & $\begin{array}{l}\text { Inquérito por } \\
\text { questionário } \\
\text { (Questionário de } \\
\text { Autorrelato de } \\
\text { Morisky-Green) }\end{array}$ & $\begin{array}{l}\text { Adesão e } \\
\text { variáveis } \\
\text { psicológicas }\end{array}$ & $\begin{array}{l}\text { Score obtido no } \\
\text { questionário }\end{array}$ & $\begin{array}{l}49,1 \% \text { dos doentes tem uma adesão } \\
\text { alta }\end{array}$ \\
\hline Magalhães et al. ${ }^{42}$ & $\begin{array}{l}\text { Inquérito por } \\
\text { questionário }\end{array}$ & Adesão (taxa) & $\begin{array}{l}\text { Esquecimento de tomar, } \\
\text { pelo menos, uma dose/ } \\
\text { /semana - frequente ou } \\
\text { muito frequentemente, } \\
\text { nos últimos } 12 \text { meses }\end{array}$ & $\begin{array}{l}\text { - } 29,7 \% \text { dos doentes classificados } \\
\text { como não aderentes } \\
\text { - Aminossalicilatos tópicos, } \\
\text { diagnóstico recente da doença e } \\
\text { idade jovem, identificados como } \\
\text { preditores de não adesão à terapêutica }\end{array}$ \\
\hline Mosca et al..$^{45}$ & $\begin{array}{l}\text { Inquérito por } \\
\text { questionário (escala } \\
\text { de adesão à } \\
\text { terapêutica de } \\
\text { Morisky) }\end{array}$ & Adesão (taxa) & $\begin{array}{l}\text { Score obtido no } \\
\text { questionário }\end{array}$ & $\begin{array}{l}\text { Taxa de adesão: } 89 \% \text {, após } \\
\text { intervenção farmacêutica }\end{array}$ \\
\hline Fernandes et al. ${ }^{30}$ & $\begin{array}{l}\text { Inquérito por } \\
\text { questionário } \\
\text { (modificação da } \\
\text { escala de adesão à } \\
\text { terapêutica de } \\
\text { Morisky) }\end{array}$ & $\begin{array}{l}\text { Não-adesão } \\
\text { (taxa) }\end{array}$ & $\begin{array}{l}\text { Score obtido no } \\
\text { questionário }\end{array}$ & $\begin{array}{l}44,8 \% \text { dos doentes classificados } \\
\text { como não aderentes }\end{array}$ \\
\hline Carvalheira et al. ${ }^{38}$ & $\begin{array}{l}\text { Inquérito por } \\
\text { telefone }\end{array}$ & Adesão (taxa) & $\begin{array}{l}\text { Pontuação obtida no } \\
\text { questionário }\end{array}$ & $\begin{array}{l}\text { Fatores psicológicos e relacionados } \\
\text { com a mediação são os que mais se } \\
\text { associam à não-adesão }\end{array}$ \\
\hline
\end{tabular}

No entanto, o facto de os questionários não serem os mesmos em cada uma das doenças/condições estudadas di- ficulta a comparabilidade dos resultados obtidos. Nos estudos em que os dados sobre adesão à terapêutica foram 


\section{QUADRO II. Métodos, medidas e resultados dos estudos de adesão à terapêutica em Portugal (continuação)}

\begin{tabular}{|l|l|l|l|l}
$\begin{array}{l}\text { Referência } \\
\text { bibliográfica }\end{array}$ & $\begin{array}{c}\text { Método de recolha } \\
\text { dos dados }\end{array}$ & $\begin{array}{c}\text { Outcomes } \\
\text { avaliados }\end{array}$ & $\begin{array}{c}\text { Medida de avaliação } \\
\text { da adesão à terapêutica } \\
\text { (quando aplicável) }\end{array}$ & \multicolumn{1}{|c|}{ Principais resultados } \\
\hline Serrabulho et al.40 & $\begin{array}{l}\text { Inquérito por } \\
\text { questionário }\end{array}$ & Adesão (taxa) & $\begin{array}{l}\text { Pontuação obtida no } \\
\text { questionário }\end{array}$ & $\begin{array}{l}\text { 83\% dos jovens apresentam boa } \\
\text { adesão à insulinoterapia; cerca de dois } \\
\text { terços dos jovens apresentam boa } \\
\text { adesão a três ou quatro fatores de } \\
\text { tratamento da diabetes (alimentação, } \\
\text { atividade física, pesquisas de glicemia } \\
\text { e insulinoterapia) }\end{array}$ \\
\hline Gonçalves ${ }^{43}$ & $\begin{array}{l}\text { Inquérito por } \\
\text { questionário }\end{array}$ & Adesão (taxa) & $\begin{array}{l}\text { Score obtido no } \\
\text { questionário }\end{array}$ & $\begin{array}{l}\text { Taxa de adesão: 72\% considera-se } \\
\text { aderente ao plano terapêutico } \\
\text { proposto }\end{array}$ \\
\hline
\end{tabular}

Legenda: $\mathrm{CEAT}-\mathrm{VIH}=$ Cuestionario para la Evaluación de la Adhesión al Tratamiento Antirretroviral; MAT=medida de adesão à terapêutica; MEMS=Medication Event Monitoring System; NVS=Newest Vital Sign; MPR=Medication Possession Ratio.

obtidos pelos registos de dispensa pelos serviços farmacêuticos hospitalares, num deles ${ }^{32}$ a adesão foi avaliada pela frequência das aquisições da terapêutica antirretroviral e no outro ${ }^{34}$ pela utilização do medication possession ratio. Moita ${ }^{31}$ recorreu a cinco medidas diferentes para estimar a adesão à terapêutica e no estudo de Mendes $^{26} \mathrm{a}$ medida utilizada foi a percentagem de cumprimento das doses prescritas durante o período de monitorização.

\section{Taxas de adesão à terapêutica}

Com a exceção do estudo de Carvalheira e colaboradores, ${ }^{39}$ onde foi avaliada a descontinuação do regime terapêutico, em todos os estudos foi avaliada a componente da implementação do tratamento. Estima-se que durante o primeiro ano de tratamento mais de $50 \%$ dos doentes descontinue o seu tratamento; ${ }^{46}$ de facto, no estudo de Carvalheira e colaboradores, ${ }^{39}$ praticamente metade dos participantes descontinuou o seu tratamento e, destes, a maioria nos primeiros três meses de tratamento. O primeiro ano de tratamento é, por isso, um período crítico, no qual a decisão de continuar ou não o tratamento tende a perdurar. ${ }^{47}$ No estudo de Fernandes e colaboradores ${ }^{30}$ a avaliação foi feita pela negativa, sendo avaliada a taxa de não-adesão à terapêutica.

Relativamente aos valores de adesão à terapêutica, a utilização de diferentes escalas, como referido, não permite uma comparação de resultados. No entanto, analisando as taxas de adesão, estas variam de $41,6 \%$ no estu- do de Silva ${ }^{35}$ até aos $89 \%$ no estudo de Mosca e colaboradores. ${ }^{45}$ No estudo de Fernandes, ${ }^{34}$ apesar de a taxa de adesão corresponder a 74,1\%, a autora verificou que mais de $80 \%$ dos participantes do estudo tiveram um intervalo sem medicação superior a 30 dias. No estudo de Moreno, ${ }^{48} 44 \%$ dos doentes transplantados referiram já se terem esquecido de tomar os seus medicamentos imunossupressores. Nos estudos que avaliaram a relação entre a adesão à terapêutica e variáveis psicológicas ${ }^{24,27,33,36,41,49,51}$ verificou-se uma relação positiva entre as mesmas.

A doença/condição estudada influenciou as taxas de adesão obtidas. Analisando as duas principais doenças/condições objeto de estudo de adesão à terapêutica, na infeção pelo $\mathrm{VIH}^{24,27,32-34}$ a taxa de adesão foi consideravelmente mais elevada do que nos estudos de adesão na hipertensão arterial, não se obtendo valores superiores a 50\% nesta última, ${ }^{25,31,35-37}$ ou seja, as taxas de adesão foram mais elevadas em doenças/condições potencialmente percecionadas pelos doentes como de risco superior.

\section{DISCUSSÃO E CONCLUSÕES}

O relatório da Organização Mundial da Saúde ${ }^{1}$ sobre adesão à terapêutica nas doenças crónicas faz eco da sugestão de Haynes e colaboradores ${ }^{52}$ de que aumentar a efetividade das medidas que promovam o aumento da adesão à terapêutica poderá ter um maior impacto nos cuidados de saúde - a nível terapêutico, mas também a 
nível económico - que qualquer melhoria no tratamento médico propriamente dito. A avaliação da adesão à terapêutica torna-se, portanto, fundamental para uma melhor compreensão dos fatores relacionados com a não-adesão para habilitar, de forma eficiente, a identificação de medidas que visem a sua melhoria e, consequentemente, a melhoria dos resultados em saúde.

Com esta revisão de âmbito foram identificadas várias lacunas de conhecimento no que diz respeito à avaliação da adesão à terapêutica na população portuguesa. Desde logo, nenhuma publicação incidiu sobre a população das regiões autónomas da Madeira e dos Açores. Com a exceção do estudo de Magalhães e colaboradores, ${ }^{32}$ nenhum estudo avaliou a adesão à terapêutica na população pediátrica. Portanto, lacunas de conhecimento a serem colmatadas através de novos estudos ou da publicação de (eventuais) estudos já realizados.

Do ponto de vista metodológico, a maioria dos estudos (22) recorreu a questionários dirigidos aos doentes para a recolha dos dados sobre adesão à terapêutica, seja de forma presencial seja através do telefone. Autilização de questionários pode ser justificada pela sua simplicidade de aplicação e reduzidos custos. No entanto, é um método mais suscetível ao erro, já que os resultados podem ser facilmente distorcidos pelos doentes. Por outro lado, este é um método que não permite de forma fácil a obtenção de informação sobre um elevado número de doentes (o estudo de Margalho e colaboradores, ${ }^{27}$ estudo de maior dimensão usando este método, não chega aos 800 participantes), o que limita a extrapolação dos resultados obtidos.

Os resultados obtidos nesta revisão de âmbito apontam ainda a necessidade de uma maior uniformidade nos métodos de avaliação da adesão à terapêutica para aumentar a comparabilidade entre estudos e analisar a evolução e tendências na população portuguesa. A adesão à terapêutica tem sido até à data avaliada, em Portugal, quase exclusivamente na componente da implementação. A adesão à terapêutica é um processo dinâmico, influenciado por múltiplos fatores em diferentes momentos temporais e, como tal, a sua avaliação transversal e descrição num único número ou taxa ou percentagem corresponde a uma simplificação de uma realidade complexa, com pouco impacto na definição de programas de intervenção nos padrões de utilização dos tratamentos. Os fatores que influenciam a decisão de iniciar um tratamento não são necessariamente os mesmos que determinam a sua (eventual) descontinuação.
Nesse sentido, os resultados deste estudo indicam uma lacuna na investigação sobre os fatores que contribuem para a decisão de iniciar um (novo) tratamento, bem como sobre os fatores que influenciam a decisão de interromper ou descontinuar esse tratamento.

Uma conclusão importante no estudo de Sousa ${ }^{49}$ é a de que quanto mais anos de acompanhamento médico do utente pelo médico de família melhor é a adesão terapêutica.

Adicionalmente, os resultados deste estudo apontam para a pertinência da utilização de fontes de informação consideradas mais robustas, como são as bases de dados de prescrição e faturação, que permitem suprimir algumas das lacunas identificadas neste estudo, desde o período de recolha de dados insuficiente até à dimensão das amostras em estudo (exceção ao estudo de Moita). ${ }^{31}$ A utilização de bases de dados constitui uma forma relativamente simples, rápida e pouco dispendiosa de reunir informação sobre o padrão de utilização de medicamentos a nível populacional. Embora o estudo da adesão à terapêutica com recurso a bases de dados eletrónicas apresente algumas limitações, como, por exemplo, a incapacidade de garantir que o doente efetivamente tomou o medicamento levantado na farmácia, as grandes bases de dados revelam-se particularmente úteis na avaliação da adesão às classes terapêuticas indicadas para os tratamentos crónicos. Quando não exista a possibilidade de adquirir os medicamentos a partir de outras fontes não capturadas na base de dados, a especificidade desta metodologia para detetar os doentes que não tomam os medicamentos prescritos é, efetivamente, muito alta. ${ }^{12}$

Apenas um dos estudos recorre a monitores eletrónicos de medicação e outro a bases de dados de prescrição e levantamento de medicação nas farmácias, métodos considerados como gold standard na investigação da componente da implementação e iniciação/descontinuação, respetivamente. ${ }^{53}$ Num momento em que a prescrição eletrónica de medicamentos é obrigatória em Portugal e o processo de conferência das receitas médicas se encontra centralizado num centro nacional de conferências de faturas, esta deverá ser nos próximos tempos uma das principais fontes de informação na investigação da adesão à terapêutica.

Finalmente, a investigação sobre adesão à terapêutica não deverá cingir-se a doentes já em tratamento, avaliando a sua execução/implementação do mesmo, mas 


\section{dever-se-á alargar a novos doentes, identificando even- tuais fatores de risco para a não-adesão primária.}

\section{REFERÊNCIAS BIBLIOGRÁFICAS}

1. Sabate E. Adherence to long-term therapies: evidence for action [Internet]. Geneva: World Health Organization; 2003. Available from: http://www. who.int/chp/knowledge/publications/adherence_full_report.pdf

2. Vrijens B, De Geest S, Hughes DA, Przemyslaw K, Demonceau J, Ruppar T, et al. A new taxonomy for describing and defining adherence to medications. Br J Clin Pharmacol. 2012;73(5):691-705.

3. Osterberg L, Blaschke T. Adherence to medication. N Engl J Med. 2005;353(5):487-97.

4. Cutler DM, Everett $W$. Thinking outside the pillbox: medication adherence as a priority for health care reform. N Engl J Med. 2010;362(17):1553-5.

5. Cramer JA, Roy A, Burrell A, Fairchild CJ, Fuldeore MJ, Ollendorf DA, et al. Medication compliance and persistence: terminology and definitions. Value Health. 2008;11(1):44-7.

6. Bugalho A, Carneiro AV. Intervenções para aumentar a adesão terapêutica em patologias crónicas. Lisboa Centro de Estudos de Medicina Baseada na Evidência, Faculdade de Medicina de Lisboa; 2004.

7. Munger MA, Van Tassell BW, LaFleur J. Medication nonadherence: an unrecognized cardiovascular risk factor. MedGenMed. 2007;9(3):58.

8. Boswell KA, Cook CL, Burch SP, Eaddy MT, Cantrell CR. Associating medication adherence with improved outcomes: a systematic literature review. Am J Pharm Benefits. 2012;4(4):e97-e108.

9. Blaschke TF, Osterberg L, Vrijens B, Urquhart J. Adherence to medications: insights arising from studies on the unreliable link between prescribed and actual drug dosing histories. Annu Rev Pharmacol Toxicol. 2012;52:275-301.

10. Kardas $P$, on behalf of $A B C$ Project Team. Ascertaining barriers for compliance: policies for safe, effective and cost-effective use of medicines in Europe [Internet]. Lodz: Medical University of Lodz; 2012 [cited 2014 Nov 23]. Available from: http://abcproject.eu/img/ABC\%20Final.pdf

11. Raebel MA, Schmittdiel J, Karter AJ, Konieczny JL, Steiner JF. Standardizing terminology and definitions of medication adherence and persistence in research employing electronic databases. Med Care. 2013;51(8 Suppl 3):S11-21.

12. Andrade SE, Kahler KH, Frech F, Chan KA. Methods for evaluation of medication adherence and persistence using automated databases. Pharmacoepidemiol Drug Saf. 2006;15(8):565-74.

13. Halpern MT, Khan ZM, Schmier JK, Burnier M, Caro JJ, Cramer J, et al. Recommendations for evaluating compliance and persistence with hypertension therapy using retrospective data. Hypertension. 2006;47(6):1039-48.

14. Urquhart J, Vrijens B. New findings about patient adherence to prescribed drug dosing regimens: an introduction to pharmionics. Eur J Hosp Pharm Sci. 2005;11(5):103-6.

15. Centre for Reviews and Dissemination. Systematic reviews: CRD's guidance for undertaking reviews in health care [Internet]. York: CRD, University of York; 2008. Available from: https://www.york.ac.uk/media/crd/Systematic_Reviews.pdf

16. Armstrong R, Hall BJ, Doyle J, Waters E. Cochrane update: 'scoping the scope' of a cochrane review. J Public Health (Oxf). 2011;33(1):147-50.

17. Brien SE, Lorenzetti DL, Lewis S, Kennedy J, Ghali WA. Overview of a formal scoping review on health system report cards. Implement Sci. 2010;5:2.

18. Arksey H, O'Malley L. Scoping studies: towards a methodological framework. Int J Soc Res Methodol. 2005;8(1):19-32.

19. Reis AC, Guerra MN, Lencastre LM. Treatment adherence and subjective wellbeing in HIV/AIDS infection. AIDS Care. 2013;25(12):1604-11.

20. Morgado M, Rolo S, Macedo AF, Pereira L, Castelo-Branco M. Predictors of uncontrolled hypertension and antihypertensive medication nonadherence. J Cardiovasc Dis Res. 2010;1(4):196-202.

21. Morgado M, Rolo S. Factors influencing medication adherence and hypertension management revisited: recent insights from cancer survivors. Hypertens Res. 2012;35(9):894-6.

22. Morgado M, Rolo S, Castelo-Branco M. Pharmacist intervention program to enhance hypertension control: a randomised controlled trial. Int J Clin Pharm. 2011;33(1):132-40.

23. Viana M, Laszczynska O, Mendes S, Friões F, Lourenço P, Bettencourt P, et al. Medication adherence to specific drug classes in chronic heart failure. J Manag Care Spec Pharm. 2014;20(10):1018-26.

24. Reis AC. Avaliação da adesão terapêutica na infeção VIH/SIDA e compreensão de variáveis psicológicas associadas [Dissertation]. Porto: Faculdade de Psicologia e de Ciências da Educação, Universidade do Porto; 2012.

25. Morgado MA. Desenvolvimento e avaliação de estratégias para aumentar a adesão à terapêutica farmacológica anti-hipertensora: estudo da intervenção do farmacêutico hospitalar no controlo da pressão arterial [Dissertation]. Covilhã: Faculdade de Ciências da Saúde, Universidade da Beira Interior; 2011.

26. Mendes SM. Padrões de adesão à terapêutica farmacológica na insuficiência cardíaca crónica [Dissertation]. Porto: Faculdade de Medicina, Universidade do Porto; 2013

27. Margalho R, Pereira M, Ouakinin S, Canavarro MC. Adesão à HAART, qualidade de vida e sintomatologia psicopatológica em doentes infectados pelo $\mathrm{VIH} / \mathrm{SIDA}$ [Adherence to HAART, quality of life and psychopathological symptoms among HIV/AIDS infected patients]. Acta Med Port. 2011;24 Suppl 2:539-48. Portuguese

28. Chin A, Alves M, Martins N, Pedro C, Ferreira A, Barbeiro C, et al. Influência dos factores financeiros no cumprimento da medicação [Influence of financial factors on adherence to medication]. Rev Port Med Geral Fam. 2012;28(5):368-74. Portuguese

29. Monterroso L, Pierdevara L, Joaquim N. Avaliação da adesão regime terapêutico dos utentes seguidos na consulta externa de psiquiatria do Centro Hospitalar Barlavento Algarvio. Rev Port Enferm Saúde Mental. 2012;7:39-45.

30. Fernandes M, Leite A, Basto M, Nobre MA, Vieira N, Fernandes R, et al. Nonadherence to antibiotic therapy in patients visiting community pharmacies. Int J Clin Pharm. 2014;36(1):86-91.

31. Moita BR. Adesão terapêutica e hipertensão arterial: o uso de fontes administrativas de dados de prescrição e levantamento de medicação na estimação da adesão à terapêutica com antagonistas dos recetores da angiotensina [Dissertation]. Lisboa: Escola Nacional de Saúde Pública, Universidade Nova de Lisboa; 2012

32. Lisboa CA. Adesão à terapêutica em utentes infectados pelo VIH [Dissertation]. Porto: Faculdade de Farmácia, Universidade do Porto; 2009.

33. Reis AC. Adesão terapêutica na infeção pelo vírus da imunodeficiência humana [Dissertation]. Porto: Faculdade de Psicologia e de Ciências da Educação, Universidade do Porto; 2007.

34. Fernandes MC. Adherence to antiretroviral treatment in HIV-1 infected subjects: prevalence and associated factors [Dissertation]. Lisboa: Faculdade de Farmácia, Universidade de Lisboa; 2012.

35. Silva JM. Estudo da adesão à terapêutica farmacológica anti-hipertensora [Dissertation]. Covilhã: Faculdade de Ciências da Saúde, Universidade da Beira Interior; 2013.

36. Ribeiro DR. Adesão terapêutica e qualidade de vida em adultos e adultos idosos com hipertensão: fatores motivacionais [Dissertation]. Porto: Faculdade de Psicologia e de Ciências da Educação, Universidade do Porto; 2013.

37. Pinto AP, José HM. Hypertension and adherence to the therapeutic regimen 
in primary health care. J Nurs UFPE. 2012;6(7):1638-47.

38. Carvalheira A, Forjaz V, Pereira NM. Adherence to phosphodiesterase type 5 inhibitors in the treatment of erectile dysfunction in long-term users: how do men use the inhibitors? Sex Med. 2014;2(2):96-102.

39. Carvalheira AA, Pereira NM, Maroco J, Forjaz V. Dropout in the treatment of erectile dysfunction with PDE5: a study on predictors and a qualitative analysis of reasons for discontinuation. J Sex Med. 2012;9(9):2361-9.

40. Serrabulho L, Matos MG, Nabais JV, Raposo JF.A satisfação com a vida e a adesão ao tratamento da diabetes dos jovens adultos com diabetes tipo 1 [Satisfaction with life and adherence to diabetes treatment of young adults with type 1 diabetes]. Rev Port Endocrinol Diabetes Metab. 2014;9(2):122-8. Portuguese

41. Sousa MR, Peixoto MJ, Martins T. Satisfação do doente diabético com os cuidados de enfermagem: influência na adesão ao regime terapêutico [Diabetic's satisfaction with the nursing care: the influence in adherence to the therapeutic regime]. Rev Enferm Referência. 2008;II(8):59-67. Portuguese

42. Magalhães J, Dias de Castro F, Boal Carvalho P, Leite S, Moreira MJ, Cotter J. Treatment of inflammatory bowel disease: is your patient at risk of nonadherence? Acta Med Port. 2014;27(5):576-80.

43. Gonçalves MN. Comportamentos de adesão à terapêutica em pessoas com doença respiratória crónica em Portugal [Dissertation]. Lisboa: ISCTE-IUL; 2014.

44. Salgado TM, Ramos SB, Sobreira C, Canas R, Cunha I, Benrimoj SI, et al. Newest vital sign as a proxy for medication adherence in older adults. J Am Pharm Assoc (2003). 2013;53(6):611-7.

45. Mosca C, Castel-Branco MM, Ribeiro-Rama AC, Caramona MM, FernandezLlimos F, Figueiredo IV. Assessing the impact of multi-compartment compliance aids on clinical outcomes in the elderly: a pilot study. Int J Clin Pharm. 2014;36(1):98-104.

46. Vrijens B, Vincze G, Kristanto P, Urquhart J, Burnier M. Adherence to prescribed antihypertensive drug treatments: longitudinal study of electronically compiled dosing histories. BMJ. 2008;336(7653):1114-7.

47. Van Wijk BL, Klungel OH, Heerdink ER, de Boer A. Rate and determinants of 10-year persistence with antihypertensive drugs. J Hypertens. 2005;23 (11):_2101-7.
48. Moreno MF. Adesão terapêutica em doentes submetidos a transplante hepático e renal [Dissertation]. Lisboa: Escola Nacional de Saúde Pública, Universidade Nova de Lisboa; 2012.

49. Sousa JA. Aliança terapêutica em contextos de saúde: sua relação com a adesão terapêutica e com as crenças dos utentes face aos médicos e medicina [Dissertation]. Braga: Instituto de Educação e Psicologia, Universidade do Minho; 2009.

50. Sousa S, Pires A, Conceição C, Nascimento T, Grenha A, Braz L. Polimedicação em doentes idosos: adesão à terapêutica [Polypharmacy in elderly patients: medication adherence]. Rev Port Clin Geral. 2011;27(2):176-82. Portuguese

51. Costa JF. Variáveis psicológicas na adesão terapêutica em lesionados medulares [Dissertation]. Coimbra: Faculdade de Psicologia e de Ciências da Educação, Universidade de Coimbra; 2012.

52. Haynes RB, Montague P, Oliver T, McKibbon KA, Brouwers MC, Kanani R. Interventions for helping patients to follow prescriptions for medications. Cochrane Database Syst Rev. 2000;(2):CD000011.

53. Vrijens B, Heidbuchel H. Non-vitamin K antagonist oral anticoagulants: considerations on once- vs. twice-daily regimens and their potential impact on medication adherence. Europace. 2015;17(4):514-23.

\section{CONFLITOS DE INTERESSE}

Os autores declaram não possuir qualquer tipo de conflito de interesses relativamente ao presente manuscrito.

\author{
ENDEREÇO PARA CORRESPONDÊNCIA \\ André Coelho \\ Escola Superior de Tecnologia da Saúde de Lisboa \\ Av. D. João II, Lote 4.69 .01 \\ 1990-096 Lisboa \\ E-mail: andre.coelho@estesl.ipl.pt
}

Recebido em 04-07-2015

Aceite para publicação em 01-08-2017

\section{ABSTRACT}

\section{RESEARCH ON MEDICATION ADHERENCE IN THE PORTUGUESE POPULATION: A SCOPING REVIEW}

Aim: To conduct a structured literature review describing the scope (quantity, focus, and nature) of published original research on medication adherence in the Portuguese population.

Data sources: Studies on medication adherence were searched on MEDLINE, Web of Science, SciELO, and B-on.

Methods: The scoping review was conducted in December 2014 using the data sources, searching for studies on medication adherence in the Portuguese population, conducted after 2005, and/or published until the end of 2014.

Results: We identified 82 publications and 26 were selected for this review. Most studies were conducted on populations in the North and in the Lisbon and Tagus Valley Region. There were no studies on initiation of treatment and only one study on discontinuation. There was variability on the methods used for data collection and measures used for evaluation of adherence. Questionnaires were the main source of information. Adherence rates varied from $41.6 \%$ to $89 \%$, depending on the disease or condition studied.

Conclusions: The different methods used for data collection and evaluation of adherence, the short periods of time for data collection, and the exclusive analysis of the implementation of treatment disregarding the factors that determine initiation and discontinuation of treatment, hinder the study of medication adherence in the Portuguese population using recently published research.

Keywords: Medication adherence; Review; Portugal 